\title{
Hydrodynamics of active polar systems in a (Visco)elastic background
}

\author{
Harald Pleiner $^{1}$ • Daniel Svenšek ${ }^{2}$ · Helmut R. Brand ${ }^{1,3}$
}

Received: 3 June 2016 / Revised: 14 July 2016 / Accepted: 25 July 2016 / Published online: 19 August 2016

(C) The Author(s) 2016. This article is published with open access at Springerlink.com

\begin{abstract}
We derive the full set of macroscopic equations necessary to describe the dynamics of systems with active polar order in a viscoelastic or elastic background. The active polar order is manifested by a second velocity, whose non-zero modulus is the polar order parameter and whose direction is the polar preferred direction. Viscoelasticity is described by a relaxing strain field allowing for a straightforward change from transient to permanent elasticity. Relative rotations of the elastic structure with respect to the polar direction are taken into account. The intricate coupling between active polar order and (transient) elasticity leads to a combined relaxation of the polar order parameter and the strains. The rather involved sound spectrum contains a specific excitation due to a reversible coupling between elasticity and polar order. Effects of chirality are also considered.
\end{abstract}

Keywords Liquid crystal · Macroscopic theory $\cdot$ Gel · Viscoelasticity

Harald Pleiner

pleiner@mpip-mainz.mpg.de

1 Max Planck Institute for Polymer Research, 55021 Mainz, Germany

2 Department of Physics, Faculty of Mathematics and Physics, University of Ljubljana, 1000 Ljubljana, Slovenia

3 Theoretische Physik III, Universität Bayreuth, 95440 Bayreuth, Germany

\section{Introduction}

The collective dynamics of active systems has attracted increasing attention of the physics community over the last few years (Marchetti et al. 2013). Here, we focus on systems, where the motion of the units creates an orientational order ("dynamic order"). Among them are schools of fish or flocks of birds (Katz et al. 2011; Buhl et al. 2006; Lukeman et al. 2010; Parrish and Edelstein-Keshet 1999; Ballerini et al. 2008), pattern forming growing bacteria (e.g., Proteus mirabilis) (Cisneros et al. 2007; Loose et al. 2008; Zhang et al. 2010; Fu et al. 2012; Watanabe et al. 2002; Moriyama and Matsushita 1995; Yamazaki et al. 2005; Matsushita et al. 1999, 1998), biological motors (myosin and actin) (Schaller et al. 2010, 2011; Aditi Simha and Ramaswamy 2002; Hatwalne et al. 2004; Surrey et al. 2001; Nedelec et al. 1997), or suitable suspensions of active particles.

These are non-equilibrium systems due to some internal or metabolic chemical reactions that provide the driving forces. They are called active, because they are driven individually in contrast to conventional driven systems, where an external force is applied globally (Cross and Hohenberg 1993). If the internal driving force vanishes, the active systems become passive, with no motion and no orientational order (Akhmediev et al. 2013). We will concentrate in the following on the hydrodynamics of the active state, which can be described rather universally, while the transition to the passive state requires the knowledge (or a model) of the specific driving mechanism (for a recent example cf. Svenšek et al. 2013). We only consider the polar case, where the active motion and order define a preferred direction that allows for the discrimination between front and rear or backward and forward. 
Often the active motion takes place within a passive environment. For a simple fluid background, the hydrodynamic description of active dynamic polar order has been given recently (Brand et al. 2013) (for the case of axial order cf. Brand et al. 2011). In this paper, we consider the case of a viscoelastic or a gel-like background that is relevant for growth or motion of many bacteria and for molecular motor dynamics. We investigate theoretically, in particular, how the elastic degrees of freedom interact with the active ones.

The active dynamic order should not be mixed up with orientational order in equilibrium systems, like nematic and polar nematic liquid crystals. There, a spontaneous breaking of rotational symmetry leads to a spatial structure that is not related to any external or internal field, nor to any macroscopic motion or movement of the molecules. It is described by a director (or equivalently by a second rank orientational tensor de Gennes 1975) or by a polar vector (Brand et al. 2006, 2009) for the non-polar and polar case, respectively. These quantities are static in the sense that they do not change under time reversal. This is in contrast to the active dynamic order, where in the polar case the velocity of the active entities is used. We will see that there are characteristic differences in the hydrodynamics between systems with static order versus systems with dynamic order.

The macroscopic dynamics of active polar systems in viscoelastic gels contains four key elements. First, there is the active polar ordering that gives rise to an active velocity relative to the passive one. This acts as the order parameter and defines the polar preferred direction (Section "Active polar dynamic order"). Second, in "Active transport and stress" section, we discuss the two-fluid aspects, like active polar advection and convection, as well as general aspects of the implementation of the driving force ("active stresses"). Third, the viscoelasticity of the passive background is described in "Viscoelasticity" section by a relaxing Eulerian strain field. In contrast to the more common approach of an ever more complicated phenomenological constitutive equation for the stress tensor, our description allows for a systematic and thermodynamically direct coupling of viscoelasticity with dynamic active polar order. Fourth, systems that exhibit permanent and/or relaxing elasticity and have additionally an orientational degree of order are known to allow for relative rotations, a concept that we discuss for the active polar case in "Relative rotations" section.

In "Macroscopic equations" section, we present in detail the macroscopic dynamic equations for achiral active polar systems with a viscoelastic background. We discuss in more detail the combined relaxations of elasticity, relative rotations, and active polar order in "Combined relaxations" section and the rather involved sound spectrum in "Sound spectrum" section. The paper closes with conclusions and perspective in "Conclusions and perspective" section. Additional features relevant for the chiral case are summarized in Appendix A, and in Appendix B, we compare the results with the case of crystals, for which the displacement field is a truly hydrodynamic variable.

\section{Key aspects}

\section{Active polar dynamic order}

The motion of the active entities is described by the macroscopic velocity $\boldsymbol{v}_{1}$. The passive background gives rise to another velocity $\boldsymbol{v}_{2}$. The kinetic energy, therefore, can be written as

$\epsilon_{k i n}=\frac{1}{2} \rho_{1} \boldsymbol{v}_{1}^{2}+\frac{1}{2} \rho_{2} \boldsymbol{v}_{2}^{2}=\frac{1}{2 \rho} \boldsymbol{g}^{2}+\frac{1}{2} \alpha \boldsymbol{F}^{2}$

with $\alpha=\rho_{1} \rho_{2} / \rho=\phi(1-\phi) \rho$, where $\rho_{1}=\phi \rho$ and $\rho_{2}=$ $(1-\phi) \rho$ are the mass densities of the active and passive part, respectively, with $\rho=\rho_{1}+\rho_{2}$ the total mass density and $\phi$ the active mass fraction. Accordingly, $g=\rho \boldsymbol{v}$ is the total momentum density defining the mean velocity $\boldsymbol{v}$. Of the relative velocity $\boldsymbol{F}=\boldsymbol{v}_{1}-\boldsymbol{v}_{2}$ only its modulus enters (1), since $\boldsymbol{F}^{2}=F^{2}$. The orientation of $\boldsymbol{F}$, described by the unit vector $\boldsymbol{f}=\boldsymbol{F} / F$, is not energetically fixed and describes the spontaneous breaking of rotational symmetry due to the ordered motion of the active entities. This is the appropriate hydrodynamic variable to describe polar dynamic order. Note that it is a true velocity, e.g., it changes sign under time reversal, opposite to the polarization that is used in the static polar case (Brand et al. 2006).

In contrast to a passive two-fluid description (Pleiner and Harden 2003), with an equilibrium state $g=0=F$, the active state is characterized by a finite $F>0$ due to the active motion. In particular, we assume a stationary state $F_{s}=$ const. $>0$ (and $\boldsymbol{g}=0$ ) as the basic state. It has a finite, non-vanishing active velocity $\boldsymbol{F}_{s}=F_{s} \boldsymbol{f}$. In the co-moving frame, there is $\boldsymbol{v}=0, \boldsymbol{v}_{1}=(1-\phi) F_{s} \boldsymbol{f}$, and $\boldsymbol{v}_{2}=-\phi F_{s} \boldsymbol{f}$.

Generally, there can be multiple stationary states that might depend on space, or even non-stationary, time dependent ones, but we will not deal with these cases here. We will also not consider a possible transition $F_{S} \rightarrow 0$ to the passive state (cf., e.g., Vicsek et al. 1995; Svenšek et al. 2013).

The existence of a finite active velocity is due to some internal, very often chemical driving force that, e.g., is described by some nonlinear diffusion-reaction equations. This part of the dynamics is system-specific, while we concentrate on the universal aspects. Therefore, we take the finite $F_{S}$ as given, which is technically achieved by an energetic coupling (Brand et al. 2013)

$\epsilon_{a c t}=-\boldsymbol{F}^{a c t} \cdot \boldsymbol{F}=-\alpha F_{s} F$ 
to a fictional "active force density" $\boldsymbol{F}^{a c t}=\alpha F_{s} f$. Since $\epsilon_{\text {kin }}$ and $\epsilon_{\text {act }}$ are the only parts of the total energy density $\epsilon$ that contain $F$, the conjugate is

$m \equiv \frac{\partial \epsilon}{\partial F}=\alpha\left(F-F_{S}\right)$

and vanishes in the stationary state. A dissipative coupling $\dot{F} \sim-\xi^{\prime} m$ describes the relaxation of $F$ to $F_{s}$.

\section{Active transport and stress}

In an Eulerian description, a temporal change of a variable (a field in space and time) can be caused by the advection of that quantity in a velocity field. For vectorial and tensorial fields also convection takes place. As an example, we take the concentration dynamics, which is generally of the form (Pleiner and Brand 1996)

$\dot{\phi}+v_{i} \nabla_{i} \phi+\rho^{-1} \nabla_{i} j_{i}^{\phi}=0$

with the phenomenological current $j_{i}^{\phi}$.

The advection contribution is reversible, since it shows the same time reversal behavior as $\dot{\phi}$. In the entropy production, it is compensated (together with other transport contributions) by the static isotropic pressure in the diagonal part of the stress tensor (Pleiner and Brand 1996). In a usual 1-fluid system, this zero entropy requirement is sufficient to fix this term and no material dependence is possible.

In a two-fluid system, it is not clear a priori, which velocity has to be used for advection and the zero entropy production requirement is not enough to decide this question. A reasonable procedure (Pleiner and Harden 2003) is to use the mean velocity for all transport terms, since in that case the zero entropy requirement is fulfilled. Nevertheless, the actual advection velocities can be different due to the phenomenological currents. In particular, $j_{i}^{\phi}$ contains a contribution (for the full expression cf. "Macroscopic equations" section)

$j_{i}^{\phi, R 1}=\gamma_{\|} \rho \phi(1-\phi)\left(F_{i}-F_{S} f_{i}\right)$

with the phenomenological reversible transport parameter $\gamma_{\|}$that leads to the effective, material-dependent transport velocity $\boldsymbol{v}_{t r}=\boldsymbol{v}+\gamma_{\|}(1-2 \phi)\left(\boldsymbol{F}-F_{S} \boldsymbol{f}\right)$. In the stationary state, this velocity vanishes in the co-moving frame. In a laboratory frame, on the other hand, where the active velocity is $\boldsymbol{v}_{1}=F_{s} \boldsymbol{f}$, and the passive one $\boldsymbol{v}_{2}=0$, there is a non-vanishing transport velocity $\boldsymbol{v}_{t r}=\phi F_{s} \boldsymbol{f}$ rendering the transport term a linear one. Indeed, the linearized active concentration dynamics in the laboratory frame reads

$\dot{\phi}+\phi F_{s} f_{i} \nabla_{i} \phi+\rho^{-1} \nabla_{i} j_{i}^{\phi, \prime}=0$

with $j_{i}^{\phi, \prime}$ containing the phenomenological couplings to other degrees of freedom.
For deviations from the stationary state, $\Delta \boldsymbol{v}_{1,2} \equiv \boldsymbol{v}_{1,2}-$ $\boldsymbol{v}_{1,2}^{\text {stat }}$, there is an additional, frame-independent contribution to the transport velocity in Eq. 4 given by $\Delta \boldsymbol{v}_{t r}=\gamma^{\prime} \Delta \boldsymbol{v}_{1}+$ $\gamma^{\prime \prime} \Delta \boldsymbol{v}_{2}$, with $\gamma^{\prime}=\phi+\gamma_{\|}(1-2 \phi)$ and $\gamma^{\prime \prime}=1-\phi-$ $\gamma_{\|}(1-2 \phi)$, which is material dependent, but only enters the nonlinear dynamics.

The existence of a finite velocity in the stationary state is a clear indication of the non-equilibrium situation due to the internal driving force. The active transport term reflects non-equilibrium, but, like any transport term, cannot drive the system out of equilibrium, since it is reversible with zero entropy production. In the literature (Giomi and Marchetti 2012; Maitra et al. 2014) (and in the Toner-Tu model as presented in Marchetti et al. 2013), dynamic polar order is often described by a polarization vector, $\boldsymbol{p}$, rather than by a velocity-type of variable. The active transport is thereby written as $\boldsymbol{v}+w_{1} \boldsymbol{p}$ where $\boldsymbol{v}$ is the passive velocity and $\boldsymbol{p}$ is used as the active velocity, with a phenomenological coefficient $w_{1}$. Clearly, the second part does not transform as a velocity rendering it dissipative and leading to a non-zero entropy production that drives the system. The physical origin of this additional, inadvertent driving force is dubious.

Macroscopic descriptions of active polar or nematic systems show a direct coupling of the stress tensor with the order parameter, often called "active term". Recently, we showed (Brand et al. 2014) that one cannot decide, neither from the symmetry properties of the macroscopic variables involved, nor from the structure of such a cross-coupling, whether the system studied is active or passive. Rather, that depends on whether the variables that give rise to those cross-couplings in the stress tensor are driven internally or externally or not at all. Such terms are reversible and come with counter terms that ensure zero entropy production. Therefore, they cannot drive the system. On the other hand, if the counter terms are neglected, the "active term" leads to non-zero entropy production and acts as an additional, albeit dubious driving force.

\section{Viscoelasticity}

In the rheology literature viscoelasticity is often described by an ad hoc generalization of Newton's linear relation between viscous stress and velocity gradients, involving nonlinearities, time derivatives, and more (Oldroyd 1950, 1961; Coleman and Noll 1961; Truesdell and Noll 1965; Giesekus 1966, 1982; Bird et al. 1977; Johnson and Segalman 1977, 1978; Larson 1988). Such an approach not only immediately becomes unwieldy and intractable when applied to more complicated systems, like the two-fluid and active and ordered case considered here, it is also rather opaque concerning the compliance with general thermodynamic requirements (Pleiner et al. 2005). 
Here, we will use the genuine hydrodynamic approach to describe elasticity by employing the strain tensor $\varepsilon_{i j}$ as macroscopic variable (Martin et al. 1972; Pleiner and Brand 1991). In crystals, it is related to the displacement vector $u_{i}$, the hydrodynamic variable associated with spontaneous breaking of translational symmetry, by $2 \varepsilon_{i j}=\nabla_{i} u_{j}+\nabla_{j} u_{i}$, if linearized. In general (already in crystals exhibiting transport of defects), the strain tensor is the appropriate variable. We will restrict ourselves to linear elasticity, assuming the elastic deformations caused by the active particles to be small.

The linear dynamic elastic equation reads in Eulerian description (Pleiner et al. 2004)

$\dot{\varepsilon}_{i j}-A_{i j}+v_{k} \nabla_{k} \varepsilon_{i j}+\varepsilon_{k j} \nabla_{i} v_{k}+\varepsilon_{k i} \nabla_{j} v_{k}+W_{i j}=0$

where $A_{i j}=\left(\nabla_{i} v_{j}+\nabla_{j} v_{i}\right) / 2$ is the "rate-of-strain" tensor and $W_{i j}$ a phenomenological current. For the transport terms, we have kept seemingly nonlinear expressions, since in an active system those terms have linear parts as discussed in the previous section.

For ordinary, permanent elasticity $W_{i j}$ contains diffusion-like contributions, called vacancy diffusion in crystals, which are usually neglected. For viscoelasticity or transient elasticity, the current $W_{i j}$ contains the relaxation of the strains,

$W_{i j}^{\text {relax }}=\tau_{i j k l} \Xi_{k l}$

where $\Xi_{i j}=\partial \epsilon / \partial \varepsilon_{i j}$ is the elastic stress tensor, the conjugate to the strain tensor, that is obtained as a partial derivative of the the total energy density $\epsilon$. Since $\Xi_{i j}$ contains a contribution $\sim \varepsilon_{i j}$, elastic strains vanish on a finite time scale. Further contributions to $\Xi_{i j}$, describing static couplings to other degrees of freedom, are listed in "Macroscopic equations" section.

In addition to relaxation, there are reversible and irreversible contributions to $W_{i j}$ that show in a lucid manner the dynamic couplings of the elastic degree of freedom to the other ones. Using standard hydrodynamic procedures (Pleiner and Brand 1996) for their derivation, one guarantees proper thermodynamic behavior, like positivity of entropy production for dissipative processes and zero entropy production for reversible ones. The full expression for $W_{i j}$ will be presented in Section "Macroscopic equations". Here, we only want to discuss the (reversible) seemingly nonlinear contributions

$W_{i j}^{R n l}=\beta_{7}\left(\varepsilon_{k j} \nabla_{i}+\varepsilon_{k i} \nabla_{j}\right)\left(m f_{k}\right)+\beta_{8} m f_{k} \nabla_{k} \varepsilon_{i j}$

with the phenomenological material parameters $\beta_{7}$ and $\beta_{8}$. In Eq. 7 , the advection and convective terms are written with the mean velocity $\boldsymbol{v}$, but with Eq. 9, these velocities effectively are $\boldsymbol{v}+\beta_{8} \alpha\left(\boldsymbol{F}-\boldsymbol{F}_{s}\right)$ and $\boldsymbol{v}+\beta_{7} \alpha\left(\boldsymbol{F}-\boldsymbol{F}_{s}\right)$, for advection and convection, respectively. As a result, they are material dependent. For example, the convection of elastic deformations takes place with the active velocity, $\boldsymbol{v}_{1}$, if $\beta_{7}=1 / \rho_{1}$, and with the passive one, $\boldsymbol{v}_{2}$, if $\beta_{7}=-1 / \rho_{2}$, but generally it will be something in-between. In the stationary state, which is homogeneous, the convection vanishes. For advection, there is a non-vanishing stationary velocity $\phi F_{s} f$ in the laboratory frame.

The generalization of this hydrodynamic approach to nonlinear viscoelasticity (transient elasticity) using the Eulerian strain tensor has been given in Temmen et al. (2000, 2001), Pleiner et al. (2000, 2004), and Grmela (2002), and its practical usefulness in describing many experiments has been demonstrated, recently (Müller et al. 2016a; b). An alternative way of describing viscoelasticity is based on the use of a relaxing orientational order parameter tensor (Doi and Edwards 1986; Pleiner et al. 2002) with rather equivalent results (Pleiner et al. 2004).

\section{Relative rotations}

Rotations of an elastic structure are related to the displacement vector by $\Omega_{i j}=\frac{1}{2}\left(\nabla_{i} u_{j}-\nabla_{j} u_{i}\right)$. In isotropic elastomers, this variable is irrelevant. If there is an independent preferred direction present, rotations of the preferred direction relative to the elastic medium, $\tilde{\Omega}_{i}$, are additional independent macroscopic degrees of freedom. They have been introduced for nematic liquid crystal elastomers by deGennes (1980). This concept has been included into the macroscopic dynamic description of liquid crystalline elastomers (Brand and Pleiner 1994). The nonlinear generalization of relative rotations (Menzel et al. 2007) is vital in describing and explaining the reorientation behavior of the nematic director under an external mechanical force (Menzel et al. 2009a, b; Urayama et al. 2007; Rogez and Martinoty 2011).

In the present case, the preferred direction is $f_{i}$ and

$\tilde{\Omega}_{i}=\delta f_{i}-\Omega_{i}^{\perp}=\delta f_{i}-\frac{1}{2} f_{j}\left(\nabla_{i} u_{j}-\nabla_{j} u_{i}\right)$

is perpendicular to $f_{i}$, since $f_{i} \tilde{\Omega}_{i}=0$. The dynamic equation can be written as

$\dot{\tilde{\Omega}}_{i}+v_{k} \nabla_{k} \tilde{\Omega}_{i}+Z_{i}=0$

Relative rotations are not truly hydrodynamic variables, since they are neither conserved nor related to any spontaneously broken continuous symmetry. Therefore, they relax in a finite time and the dissipative part of $Z_{i}$ contains

$Z_{i}^{\text {relax }}=\tau^{D} \delta_{i j}^{\perp} \Sigma_{j}$

where the relative torque, $\Sigma_{i}=\partial \epsilon / \partial \tilde{\Omega}_{i}$, is the conjugate to the relative rotations and is obtained as a partial derivative of the the total energy density $\epsilon$. The transverse Kronecker 
symbol, $\delta_{i j}^{\perp}=\delta_{i j}-f_{i} f_{j}$, reflects the transverse nature of $\tilde{\Omega}, \Sigma_{i}$ and $Z_{i}$.

The full expression for $Z_{i}$ will be given below. In contrast to the nematic case, $\tilde{\Omega}_{i}$ has the transformation properties of a velocity and, therefore, shows in $Z_{i}$ rather different types of couplings to the other variables.

\section{Macroscopic equations}

\section{Statics}

We are now in a position to present the full macroscopic dynamic equations for active polar order in a viscoelastic background. In addition to the variables already introduced in "Key aspects" section, total energy density $\epsilon$, active concentration $\phi$, total mass density $\rho$, total momentum density $\boldsymbol{g}$, polar order $F$ and preferred direction $f_{i}$, elastic strain tensor $\varepsilon_{i j}$, and relative rotations $\tilde{\Omega}_{i}$, there is the entropy density $\sigma$. The latter is related to all other variables by the Gibbs relation

$T d \sigma=d \epsilon-\Pi d \phi-\mu d \rho-\boldsymbol{v} \cdot d \boldsymbol{g}-m d F-h_{i} d f_{i}$

$$
-\Xi_{i j} d \varepsilon_{i j}-\Sigma_{i} d \tilde{\Omega}_{i}
$$

with the thermodynamic conjugates as prefactors, temperature $T$, osmotic pressure $\Pi$, chemical potential $\mu$, the mean velocity $\boldsymbol{v}$, the order conjugate $m$, the 'molecular field' $h_{i}$, the elastic stress tensor $\Xi_{i j}$, and the relative torque $\Sigma_{i}$. They all follow from the total energy density as partial derivatives. Note that $h_{i}$ and $\Sigma_{i}$ have to be transverse $\left(f_{i} h_{i}=0=f_{i} \Sigma_{i}\right)$ and $\Xi_{i j}=\Xi_{j i}$ symmetric.

In the absence of any orienting field, the orientation of $f_{i}$ is energetically not fixed and only $\nabla_{j} f_{i}$ enters the Gibbs relation. It is therefore useful to make this explicit in the Gibbs relation

$$
\begin{aligned}
d \epsilon= & T d \sigma+\Pi d \phi+\mu d \rho+\boldsymbol{v} \cdot d \boldsymbol{g}+m^{\prime} d F+h_{i}^{\prime} d f_{i} \\
& +\Psi_{i j} d \nabla_{j} F_{i}+\Xi_{i j} d \varepsilon_{i j}+\Sigma_{i} d \tilde{\Omega}_{i}
\end{aligned}
$$

with $h_{i}=h_{i}^{\prime}-F \nabla_{j} \Psi_{i j}$ and $m=m^{\prime}-f_{i} \nabla_{j} \Psi_{i j}$.

To guarantee rotational invariance of the energy, there must be the symmetry

$$
\begin{aligned}
& h_{i}^{\prime} \delta f_{j}+\Psi_{k i} \nabla_{j} F_{k}+\Psi_{i k} \nabla_{k} F_{j}+2 \Xi_{i k} \varepsilon_{j k}+\Sigma_{i} \tilde{\Omega}_{j} \\
& \quad=\{i \Longleftrightarrow j\}
\end{aligned}
$$

To be meaningful, we need a definite expression for the energy density

$\epsilon=\epsilon_{\text {kin }}+\epsilon_{\text {act }}+\epsilon_{\text {grad }}+\epsilon_{\text {state }}+\epsilon_{\text {mix }}+\epsilon_{\text {elast }}+\epsilon_{\text {rot }}$
Restricting ourselves to the harmonic approximation, symmetry allows for the expressions

$$
\begin{aligned}
\epsilon_{\text {grad }}= & \frac{1}{2} K_{i j k l}\left(\nabla_{j} F_{i}\right)\left(\nabla_{l} F_{k}\right) \\
\epsilon_{\text {state }}= & \frac{1}{2} c_{\rho \rho}(\delta \rho)^{2}+\frac{1}{2} c_{\sigma \sigma}(\delta \sigma)^{2}+\frac{1}{2} c_{\phi \phi}(\delta \phi)(\delta \phi) \\
& +c_{\rho \phi}(\delta \rho)(\delta \phi)+c_{\rho \sigma}(\delta \rho)(\delta \sigma)+c_{\sigma \phi}(\delta \sigma)(\delta \phi) \\
\epsilon_{\text {mix }}= & \left(\sigma_{i j k}^{\sigma} \nabla_{k} \sigma+\sigma_{i j k}^{\rho} \nabla_{k} \rho+\sigma_{i j k}^{\phi} \nabla_{k} \phi\right)\left(\nabla_{i} F_{j}\right) \\
\epsilon_{\text {elast }}= & \frac{1}{2} c_{i j k l} \varepsilon_{i j} \varepsilon_{k l}+\left(\chi_{i j}^{\sigma} \delta \sigma+\chi_{i j}^{\rho} \delta \rho+\chi_{i j}^{\phi} \delta \phi\right) \varepsilon_{i j} \\
\epsilon_{\text {rot }}= & \frac{1}{2} D_{1} \tilde{\Omega}_{i} \tilde{\Omega}_{i}+D_{2}\left(f_{j} \delta_{i k}^{\perp}+f_{k} \delta_{i j}^{\perp}\right) \tilde{\Omega}_{i} \varepsilon_{j k} \\
& +D_{i j k l}^{F}\left(\nabla_{j} F_{i}\right)\left(\nabla_{l} \tilde{\Omega}_{k}\right)
\end{aligned}
$$

where $\epsilon_{\text {kin }}$ and $\epsilon_{\text {act }}$ have been given in Eqs. 1 and 2, respectively. The energy $\epsilon$ is not specific for active dynamic polar order and is the same as for a passive nematic elastomer (replacing $f_{i}$ by the director $n_{i}$ everywhere).

The second rank tensors are of the uniaxial symmetric form (with $\delta_{i j}^{\perp} \equiv \delta_{i j}-f_{i} f_{j}$ ), e.g.,

$\chi_{i j}=\chi_{\|} f_{i} f_{j}+\chi_{\perp} \delta_{i j}^{\perp}$,

with two generalized susceptibilities, each. The third-order tensors are odd under time reversal and spatial inversion, e.g.,

$\sigma_{i j k}^{\rho}=\sigma_{1}^{\rho} f_{i} f_{j} f_{k}+\sigma_{2}^{\rho} f_{j} \delta_{i k}^{\perp}+\sigma_{3}^{\rho}\left(f_{i} \delta_{j k}^{\perp}+f_{k} \delta_{i j}^{\perp}\right)$,

and contain three phenomenological static parameters, each. The three fourth-order tensors have different symmetries

$$
\begin{aligned}
K_{i j k l}= & \frac{1}{2} K_{1}\left(\delta_{i j}^{\perp} \delta_{k l}^{\perp}+\delta_{i l}^{\perp} \delta_{j k}^{\perp}\right)+K_{2} f_{p} \epsilon_{p i j} f_{q} \epsilon_{q k l} \\
& +K_{3} f_{l} f_{j} \delta_{i k}^{\perp}+K_{4} f_{i} f_{j} f_{k} f_{l}+K_{5} f_{i} f_{k} \delta_{j l}^{\perp} \\
& +\frac{1}{4} K_{6}\left(f_{i} f_{l} \delta_{k j}^{\perp}+f_{j} f_{k} \delta_{i l}^{\perp}+f_{i} f_{j} \delta_{k l}^{\perp}+f_{k} f_{l} \delta_{i j}^{\perp}\right)
\end{aligned}
$$

and

$$
\begin{aligned}
c_{i j k l}= & c_{1} \delta_{i j}^{\perp} \delta_{k l}^{\perp}+c_{2}\left(\delta_{i k}^{\perp} \delta_{j l}^{\perp}+\delta_{i l}^{\perp} \delta_{j k}^{\perp}\right) \\
& +c_{3} f_{i} f_{j} f_{k} f_{l}+c_{4}\left(f_{i} f_{j} \delta_{k l}^{\perp}+f_{k} f_{l} \delta_{i j}^{\perp}\right) \\
& +c_{5}\left(f_{i} f_{k} \delta_{j l}^{\perp}+f_{i} f_{l} \delta_{j k}^{\perp}+f_{j} f_{k} \delta_{i l}^{\perp}+f_{j} f_{l} \delta_{i k}^{\perp}\right)
\end{aligned}
$$

and

$$
\begin{aligned}
D_{i j k l}^{F}= & \frac{1}{2} D_{1}^{F}\left(\delta_{i j}^{\perp} \delta_{k l}^{\perp}+\delta_{i l}^{\perp} \delta_{j k}^{\perp}\right)+D_{2}^{F} f_{p} \epsilon_{p i j} f_{q} \epsilon_{q k l} \\
& +D_{3}^{F} f_{l} f_{j} \delta_{i k}^{\perp}+\frac{1}{2} D_{4}^{F} f_{i}\left(f_{l} \delta_{k j}^{\perp}+f_{j} \delta_{k l}^{\perp}\right)
\end{aligned}
$$

containing six generalized Frank coefficients, five elastic moduli, and four mixed-type parameters, respectively. We 
do not write down here the explicit expressions for the conjugate quantities, since taking the appropriate derivatives is straightforward. We provide those expressions when necessary (e.g., in "Simple solutions" section). The order conjugate $m$ is given in Eq. 3 .

\section{Dynamic equations}

The variables introduced above follow local dynamic equations, either conservation laws or balance equations. Using the symmetry requirements, we get

$$
\begin{aligned}
& \dot{\rho}+\nabla_{i} g_{i}=0 \\
& \dot{\sigma}+\nabla_{i}\left(\sigma v_{i}\right)+\nabla_{i} j_{i}^{\sigma}=\frac{R}{T} \\
& \dot{g}_{i}+\nabla_{j}\left(v_{j} g_{i}+\delta_{i j} p+\frac{1}{2} \Psi_{j k} \nabla_{k} F_{i}+\frac{1}{2} \Psi_{i k} \nabla_{k} F_{j}\right. \\
& \left.\quad \quad \quad \Xi_{i j}+\Xi_{k j} \varepsilon_{i k}+\Xi_{k i} \varepsilon_{j k}\right)+\nabla_{j} \sigma_{i j}=0 \\
& \quad \dot{F}+v_{i} \nabla_{i} F+X=0 \\
& \dot{f}_{i}+v_{j} \nabla_{j} f_{i}+f_{j} \omega_{i j}+Y_{i}=0
\end{aligned}
$$

with the vorticity tensor $\omega_{i j}=\frac{1}{2}\left(\nabla_{i} v_{j}-\nabla_{j} v_{i}\right)$. The dynamic equations for $\phi, \varepsilon_{i j}$, and $\tilde{\Omega}_{i}$ (with the phenomenological currents $\left.j_{i}^{\phi}, W_{i j}, \Sigma_{i}\right)$ are given in Eqs. 4, 7, and 11 . These phenomenological currents, and the other ones, $j_{i}^{\sigma}$, $\Xi_{i j}, X$, and $Y_{i}$, can all be split into a reversible part (superscript $R$ ) and a dissipative part (superscript $D$ ) according to their time reversal behavior.

As discussed in "Active transport and stress" section, we have written the transport terms using the mean velocity, while additions are provided by the reversible parts of the phenomenological currents given below. For the origin of the rather lengthy non-phenomenological expression in brackets in Eq. 29 cf. Pleiner et al. $(1996,2004)$. The dynamic equation for $\boldsymbol{F}$ is $\dot{F}_{i}+v_{j} \nabla_{j} F_{i}-F_{j} \omega_{i j}+X_{i}=0$ with $X_{i}=f_{i} X+F Y_{i}$.

The source term in Eq. 28, the entropy production $R / T$, is zero for reversible processes and positive for dissipative ones. With the help of the Gibbs relation, we get for $R$ as a bilinear function of dissipative currents and generalized forces

$$
\begin{aligned}
\int R_{\sigma} d V= & \int d V\left(-j_{i}^{\sigma, D} \nabla_{i} T-j_{i}^{\phi, D} \nabla_{i}(\Pi / \rho)-\sigma_{i j}^{D} A_{i j}\right. \\
& \left.+Y_{i}^{D} h_{i}+X^{D} m+W_{i j}^{D} \Xi_{i j}+Z_{i}^{D} \Sigma_{i}\right)>0(32)
\end{aligned}
$$

Within linear irreversible thermodynamics, currents, and forces are linearly related, which allows us to use the dissipation function, $R$, written as a harmonic function of the forces alone, as a potential. In particular, using symmetry arguments again, we find

$$
\begin{aligned}
2 R= & \kappa_{i j}\left(\nabla_{i} T\right)\left(\nabla_{j} T\right)+D_{i j}\left(\nabla_{i} \Pi^{\prime}\right)\left(\nabla_{j} \Pi^{\prime}\right) \\
& +\xi_{i j k l p q}\left(\nabla_{k} \Xi_{i p}\right)\left(\nabla_{l} \Xi_{j q}\right)+\tau^{D} \delta_{i j}^{\perp} \Sigma_{i} \Sigma_{j}+b^{D} \delta_{i j}^{\perp} h_{i} h_{j} \\
& +v_{i j k l} A_{i j} A_{k l}+\xi^{\prime} m^{2}+2 \mu_{i j k}^{T} A_{i j} \nabla_{k} T \\
& +2 \mu_{i j k}^{\Pi} A_{i j} \nabla_{k} \Pi^{\prime}+2 \mu_{i j k l m}^{\Xi} A_{i j} \nabla_{l} \Xi_{k m} \\
& +2 D_{i j}^{T}\left(\nabla_{i} \Pi^{\prime}\right)\left(\nabla_{j} T\right)+2 \xi_{i j k l}^{T}\left(\nabla_{i} T\right)\left(\nabla_{k} \Xi_{j l}\right) \\
& +2 \xi_{i j k l}^{\Pi}\left(\nabla_{i} \Pi^{\prime}\right)\left(\nabla_{k} \Xi_{j l}\right)+2 \xi \delta_{i j}^{\perp} \Sigma_{i} h_{j} \\
& +\tau_{i j k l} \Xi_{i j} \Xi_{k l}+2 \Xi_{i j}\left(\tau_{i j}^{T} \delta T+\tau_{i j}^{\Pi} \delta \Pi^{\prime}+\tau_{i j}^{m} m\right)(33)
\end{aligned}
$$

and the dissipative currents are obtained as partial or functional derivatives, e.g., $X^{D}=\partial R / \partial m, j_{i}^{\sigma, D}=-\partial R / \partial \nabla_{i} T$ or $W_{i j}^{D}=\delta R / \delta \Xi_{i j}=\partial R / \partial \Xi_{i j}-\nabla_{k} \partial R / \partial \nabla_{k} \Xi_{i j}$ etc. We do not write down here the explicit expressions for the dissipative currents, and only provide them when necessary.

The second rank tensors have the form of Eq. 22, the third rank ones are as in Eq. 23, the fourth-order tensors $v_{i j k l}$ and $\tau_{i j k l}$ have the same form as the elasticity tensor Eq. 25 and the fourth rank tensors $\xi_{i j k l}^{T}$ and $\xi_{i j k l}^{T}$ take the form given in Eq. 24. The structure of the fifth rank tensor $\mu_{i j k l m}^{\Xi}$ and the sixth rank tensor $\xi_{i j k l p q}$ will be elucidated in Appendix B.

The dissipation function contains the relaxations of the order parameter $\left(\xi^{\prime}\right)$, of elastic strains $\left(\tau_{i j k l}\right)$, and of relative rotations $\left(\tau^{D}\right)$, already discussed in "Key aspects" section, as well as heat conduction $\left(\kappa_{i j}\right)$, active concentration diffusion $\left(D_{i j}\right)$, viscosity $\left(v_{i j k l}\right)$, and rotational viscosity of the polar direction $\left(b^{D}\right)$. There are various interesting crosscouplings, thermo-diffusion $\left(D_{i j}^{T}\right)$; thermo-strain diffusion $\left(\xi_{i j k l}^{T}\right)$; solutal-strain diffusion $\left(\xi_{i j k l}^{\Pi}\right)$; couplings of flow to temperature $\left(\mu_{i j k}^{T}\right)$, to osmotic pressure $\left(\mu_{i j k}^{\Pi}\right)$, and to strain diffusion $\left(\mu_{i j k l m}^{\Xi}\right)$; couplings of strain relaxation to temperature $\left(\tau_{i j}^{T}\right)$, to concentration $\left(\tau_{i j}^{\Pi}\right)$, and to order parameter $\left(\tau_{i j}^{m}\right)$; and a coupling between relative rotations and polar orientations $(\xi)$. The dissipative flow couplings $\left(\mu_{i j k}^{T, \Pi}\right)$ and $\left(\mu_{i j k l m}^{\Xi}\right)$ are specific for a dynamic polar system and are absent in, e.g., passive nematic elastomers. The strain diffusion $\left(\xi_{i j k l p q}\right)$ is a sixth rank tensor with 16 independent coefficients whose detailed structure will be given in Appendix B.

The reversible parts of the phenomenological currents cannot be derived from a potential. Rather, one writes down all terms that are possible by symmetry and makes sure that 
appropriate cross-coupling terms cancel each other in the entropy production

$$
\begin{gathered}
0=\int d V\left(-j_{i}^{\sigma, R} \nabla_{i} T-j_{i}^{\phi, R} \nabla_{i}(\Pi / \rho)-\sigma_{i j}^{R} A_{i j}\right. \\
\left.+Y_{i}^{R} h_{i}+X^{R} m+W_{i j}^{R} \Xi_{i j}+Z_{i}^{R} \Sigma_{i}\right)
\end{gathered}
$$

Neglecting some higher order gradient terms, we find

$$
\begin{aligned}
j_{i}^{\sigma, R}= & \beta_{\|} f_{i} m+\beta_{\perp} \delta_{i j}^{\perp} h_{j}+\beta_{\perp}^{\Omega} \delta_{i j}^{\perp} \Sigma_{j} \\
j_{i}^{\phi, R}= & \gamma_{\|} f_{i} m+\gamma_{\perp} \delta_{i j}^{\perp} h_{j}+\gamma_{\perp}^{\Omega} \delta_{i j}^{\perp} \Sigma_{j} \\
\sigma_{i j}^{R}= & a_{i j} m+\lambda_{i j k} h_{k}+\lambda_{i j k}^{\Omega} \Sigma_{k} \\
Y_{i}^{R}= & \delta_{i j}^{\perp}\left(\beta_{\perp} \nabla_{j} T+\gamma_{\perp} \nabla_{j} \Pi^{\prime}+\beta_{1} \nabla_{j} m+\beta_{k l}^{W} \nabla_{k} \Xi_{j l}\right) \\
& +\lambda_{k j i} A_{j k}+Y_{i}^{R n l} \\
X^{R}= & \beta_{\|} f_{i} \nabla_{i} T+\gamma_{\|} f_{i} \nabla_{i} \Pi^{\prime}+a_{i j} A_{i j}+\beta_{1} \delta_{i j}^{\perp} \nabla_{j} h_{i} \\
& +\beta_{1}^{\Omega} \delta_{i j}^{\perp} \nabla_{i} \Sigma_{j}+\tilde{\beta}_{k l}^{W} f_{i} \nabla_{k} \Xi_{i l}+X^{R n l} \\
Z_{i}^{R}= & \delta_{i j}^{\perp}\left(\beta_{\perp}^{\Omega} \nabla_{j} T+\gamma_{\perp}^{\Omega} \nabla_{j} \Pi^{\prime}+\beta_{1}^{\Omega} \nabla_{j} m\right) \\
& +\beta_{k l}^{\Omega} \delta_{i j}^{\perp} \nabla_{k} \Xi_{j l}+\lambda_{k j i}^{\Omega} A_{j k}+Z_{i}^{R n l} \\
W_{i j}^{R}= & \frac{1}{2}\left(\tilde{\beta}_{k j}^{W} f_{i}+\tilde{\beta}_{k i}^{W} f_{j}\right) \nabla_{k} m \\
& +\frac{1}{2}\left(\delta_{i k}^{\perp} \beta_{j l}^{W}+\delta_{j k}^{\perp} \beta_{i l}^{W}\right) \nabla_{l} h_{k} \\
& +\frac{1}{2}\left(\delta_{i k}^{\perp} \beta_{j l}^{\Omega}+\delta_{j k}^{\perp} \beta_{i l}^{\Omega}\right) \nabla_{l} \Sigma_{k}+W_{i j}^{R n l}
\end{aligned}
$$

with the second rank tensors of the form of Eq. 22, and the generalized flow alignment tensors are

$\lambda_{i j k}=\lambda\left(\delta_{i k}^{\perp} f_{j}+\delta_{j k}^{\perp} f_{i}\right)$

$\lambda_{i j k}^{\Omega}=\lambda^{\Omega}\left(\delta_{i k}^{\perp} f_{j}+\delta_{j k}^{\perp} f_{i}\right)$

Relative rotations couple reversibly to all other variables $\left(\beta_{\perp}^{\Omega}, \gamma_{\perp}^{\Omega}, \lambda_{i j k}^{\Omega}, \beta_{1}^{\Omega}, \beta_{k l}^{\Omega}\right)$, except to reorientations of the preferred direction. The latter couple to the elastic degree of freedom $\left(\beta_{k l}^{W}\right)$ as does the order parameter $\left(\beta_{1 k l}^{W}\right)$. All other terms are already present in systems with dynamic polar order in a simple fluid background (Brand et al. 2013). Among them is the so-called active term $\left(a_{i j}\right.$ in the stress term) mentioned in "Active transport and stress" section and discussed in detail in (Brand et al. 2014).

The nonlinear term $W_{i j}^{R n l}$ has been used in "Viscoelasticity" section to show that it modifies the transport velocities of the elastic strain tensor. The counter terms are $\sim \beta_{7}$ and $\sim \beta_{8}$ in Eq. 45. Similarly,

$$
\begin{aligned}
Y_{i}^{R n l}= & \beta_{3} \delta_{i k}^{\perp} f_{j}\left(\nabla_{j} F_{k}\right) m+\beta_{4} \delta_{i k}^{\perp} f_{j} \omega_{k j} m \\
X^{R n l}= & \beta_{3} \delta_{i k}^{\perp} f_{j}\left(\nabla_{j} F_{k}\right) h_{i}+\beta_{4} \delta_{i k}^{\perp} f_{j} \omega_{j k} h_{i} \\
& +2 \beta_{7} f_{k} \nabla_{i}\left(\Xi_{i j} \varepsilon_{k j}\right)-\beta_{8} \Xi_{i j} f_{k} \nabla_{k} \varepsilon_{i j} \\
& -\beta_{5} f_{k} \Sigma_{i} \nabla_{k} \tilde{\Omega}_{i} \\
Z_{i}^{R n l}= & \beta_{5} m f_{k} \nabla_{k} \tilde{\Omega}_{i}
\end{aligned}
$$

and $\beta_{5}$ adds to the advective velocity of relative rotations in Eq. 11, while $\beta_{3}$ and $\beta_{4}$ contribute to the advective and convective velocity of $f_{i}$, Eq. 31 respectively.

\section{Simple solutions}

\section{Combined relaxations}

The set of macroscopic equations derived above contains three variables that relax to a certain stationary value, even when the deviations are homogeneous in space: Elastic strains $\varepsilon_{i j}$ relax to zero, since elasticity is transient only for a visco-elastic medium, the order parameter of the active polar order $\delta F$ relaxes to its non-zero stationary value $F_{S}$, and the relative rotations $\tilde{\Omega}_{i}$ relax to zero, since they constitute a non-hydrodynamic degree of freedom.

Actually, these three types of variables are coupled and so are their relaxations. The free energy (21) shows the coupling between relative rotations and elastic strains (via $D_{2}$ ), well-known already from nematic elastomers, while the active polar order couples dynamically to elasticity via the very last term $\left(\sim \tau_{i j}^{m}\right)$ in the dissipation function (33). In addition, elastic strains are related dynamically to flow, $A_{i j}$, Eq. 7, and statically to changes of entropy or temperature, density and concentration, Eq. 20. In the homogeneous case, these variables do not have their own dynamics and are simply slaved by the strains. Thus, for homogeneous relaxations, we are left with

$$
\begin{aligned}
& \dot{\varepsilon}_{i j}+\tau_{i j k l} \Xi_{k l}+\tau_{i j}^{m}\left(F-F_{s}\right)=0 \\
& \dot{\tilde{\Omega}}_{i}+\tau^{D}\left(D_{1} \tilde{\Omega}_{i}+D_{2}\left[f_{j} \delta_{i k}^{\perp}+f_{k} \delta_{i j}^{\perp}\right] \varepsilon_{j k}\right)=0 \\
& \dot{F}+\xi^{\prime} \alpha\left(F-F_{s}\right)+\tau_{i j}^{m} \Xi_{i j}=0
\end{aligned}
$$

with $\Xi_{i j}=c_{i j k l} \varepsilon_{k l}+D_{2}\left(f_{j} \delta_{i k}^{\perp}+f_{i} \delta_{j k}^{\perp}\right) \tilde{\Omega}_{k}$.

We notice that relative rotations only couple to shear strains $\left(\varepsilon_{i j}\right.$ off-diagonal with respect to $\boldsymbol{f}$ ), while the active polar order couples to compressional strains ( $\varepsilon_{i j}$ diagonal with respect to $f$ ). The former case is also present for transient nematic elastomers (nematic polymers), although, to our knowledge, it has not been considered before. It is not related to active order and will be discussed only briefly at the end of this section. We will concentrate here on the coupling to polar active order. To reduce the algebraic complexity of the equations, and since it is a rather good approximation for polymers, we will assume incompressibility in the strains, $\varepsilon_{i i}=0$. The remaining two equations are (with $f$ along the z-direction)

$$
\begin{aligned}
& \dot{\varepsilon}_{z z}+\zeta \varepsilon_{z z}+\tau_{m} \alpha\left(F-F_{s}\right)=0 \\
& \dot{F}+\xi^{\prime} \alpha\left(F-F_{s}\right)+\tau_{m} c_{m} \varepsilon_{z z}=0
\end{aligned}
$$


with the abbreviations $\zeta \equiv \tau_{3}\left(c_{3}-c_{4}\right)+2 \tau_{4}\left(c_{4}-c_{1}-c_{2}\right)$, $c_{m} \equiv c_{1}+c_{2}+c_{3}-2 c_{4}$, and $\tau_{m} \equiv \tau_{\|}^{m}=-2 \tau_{\perp}^{m}$. The latter relation is necessary to maintain incompressibility for all times, $\dot{\varepsilon}_{i i}=0$. There are appropriate relations for combinations of $\tau$ 's and $c$ 's, which we will not show here.

The general solution is a coupled sum of two exponential decays

$$
\left\{\begin{array}{c}
\varepsilon_{z z} \\
F-F_{s}
\end{array}\right\}=\left\{\begin{array}{l}
A_{1} \\
B_{1}
\end{array}\right\} \exp \left(-\lambda_{1} t\right)+\left\{\begin{array}{l}
A_{2} \\
B_{2}
\end{array}\right\} \exp \left(-\lambda_{2} t\right)
$$

with the inverse relaxation times

$\lambda_{1,2}=\frac{1}{2}\left(\zeta+\alpha \xi^{\prime}\right) \pm \frac{1}{2}\left(\left(\zeta-\alpha \xi^{\prime}\right)^{2}+4 \alpha c_{m} \tau_{m}^{2}\right)^{1 / 2}$

Although the coupling constant $\tau_{m}$ is bound from above, $\tau_{m}^{2}<\tau_{3} \xi^{\prime}$, by the positivity requirement of the dissipation function, it is not guaranteed that the $\lambda$ 's are both positive. This is achieved by the requirement of the additional relation $\tau_{3}=-2 \tau_{4}$.

The $A$ - and $B$-amplitudes are coupled, e.g., $\alpha \tau_{m} B_{1,2}=$ $-\left(\lambda_{1,2}+\zeta\right) A_{1,2}$, and any relaxation of one variable triggers the relaxation of the other. Assuming initially $F=F_{S}$ and a finite deformation $\varepsilon_{z z}(t=0)=A$, the latter not only relaxes to zero with the amplitudes

$A_{1,2}= \pm \frac{\zeta-\lambda_{2,1}}{\lambda_{1}-\lambda_{2}} A$

but also induces a transient change in the active polar order

$B_{1}=-B_{2}=\frac{c_{m} \tau_{m}}{\lambda_{1}-\lambda_{2}} A$

starting with the slope $B\left(\lambda_{2}-\lambda_{1}\right)$ and finally relaxing to zero with the longest relaxation time. Vice versa, an initial order parameter deviation from the stationary value, $F(t=0)-F_{s}=B$ leads to the appropriate result of an induced transient compression

$A_{1}=-A_{2}=\frac{\alpha \tau_{m}}{\lambda_{1}-\lambda_{2}} B$

and $\quad B_{1,2}= \pm \frac{\alpha \xi^{\prime}-\lambda_{2,1}}{\lambda_{1}-\lambda_{2}} B$

The combined relaxation of relative rotations and shear strains, e.g. $\tilde{\Omega}_{x}$ and $\varepsilon_{x z}$, is described by the sum of two exponentials (similar to Eq. 52) with the inverse relaxation times

$\lambda_{3,4}=\tau_{5} c_{5}+\frac{\tau^{D} D_{1}}{2} \pm\left(\left[\tau_{5} c_{5}-\frac{\tau^{D} D_{1}}{2}\right]^{2}+8 \tau_{5} \tau^{D} D_{2}^{2}\right)^{1 / 2}$

again indicating that relaxing relative rotations induce shear strains and vice versa.

\section{Sound spectrum}

In the preceding section, we concentrated on homogeneously relaxing strains. Here, we will consider the case that either elasticity is permanent (the relaxation times diverge) or that experiments are done on a time scale small compared to the relaxation times. We concentrate on sound-like excitations, i.e., propagating plane waves $(\sim \exp i(\omega t-\boldsymbol{k} \cdot \boldsymbol{r}))$ characterized by a frequency $\omega$ and a wave vector $\boldsymbol{k}$. We only consider effects to order $\omega \sim k$ and neglect dissipative effects.

In simple liquids, the only propagating excitation is ordinary sound, which is based on the reversible coupling between momentum and density dynamics via the density dependence of the pressure (compressibility). It is called longitudinal, since the space and time-dependent velocity is along the wave propagation direction given by $\boldsymbol{k}$.

In nematic and polar nematic systems, there is no additional propagating excitation in order $\omega \sim k$, since the nematic order is related to broken rotational symmetry and the flow alignment coupling $\left(\lambda_{i j k}\right)$ only leads to $\omega \sim k^{2}$. With active polar order the situation is completely different (Brand et al. 2013), since the preferred direction is a second velocity. It has been shown that the reversible couplings of entropy, concentration and the stress tensor to the polar order, $\gamma_{\|}, \beta_{\|}, a_{\|}$in Eqs. 35-37, lead to a second, generally propagating, longitudinal sound. It is anisotropic, depending on the orientation of $\boldsymbol{k}$ relative to $\boldsymbol{f}$, and couples to the ordinary sound such that the latter also becomes anisotropic. In addition, it reflects the broken time reversal symmetry due to the non-equilibrium nature of the active polar state (cf. Eq. (76) of Brand et al. 2013).

In passive elastomers and crystals elasticity allows for additional propagating sound-like excitation. The direct relation between strain dynamics and flow, Eq. 7, and the elastic stress contribution to the stress tensor, Eq. 29, lead to transverse, shear-elastic sound, and an elastic contribution to the longitudinal sound velocity. Only the elastic moduli $c_{i j k l}$ in Eq. 20 are involved as material parameters.

In the present system, we have all these three excitations together, and, of course, they are coupled. In addition, there are extra excitations beyond those of the individual subsystems. In particular, the direct reversible coupling between elasticity and active polar order, provided by the reactive transport parameter $\beta_{1}^{W}$ in Eqs. 39 and 41, results in an additional propagating mode with

$\omega_{1,2}^{2}=\alpha\left(\beta_{1}^{W}\right)^{2}\left(4 c_{5} k_{\perp}^{2}+c_{3} k_{\|}^{2}\right)$

that also couples to the previously discussed modes. It involves compressional, $\varepsilon_{z z}$, and shear strains $\varepsilon_{x z}+\varepsilon_{y z}$. This mode can be weakly damped due to the relaxation of $F$. 
There are other reversible cross-coupling terms, like $\beta_{1}$ in Eqs. 38 and 39, and $\beta^{W}$ in Eqs. 38 and 41. They only contribute to the order $\omega \sim k^{2}$, since in both cases reorientations of the active polar direction are involved and $f_{i}$ is a variable associated with a spontaneously broken continuous symmetry, of which only gradients enter the free energy. In principle, also the reversible cross-couplings in Eq. $40, \lambda_{i j k}^{\Omega}, \beta_{1}^{\Omega}$, and $\beta^{\Omega}$, of relative rotations with, respectively, flow, Eq. 37, polar order, Eq. 39, and elasticity, Eq. 41, lead to sound-like excitations; in particular to $\omega^{2}=\left(D_{1} / \rho\right)\left(\lambda^{\Omega}\right)^{2} k^{2}, \omega^{2}=\alpha D_{1}\left(\beta_{1}^{\Omega}\right)^{2} k_{\perp}^{2}$ and $\omega^{2}=$ $D_{1}\left(\beta^{\Omega}\right)^{2}\left(\left[c_{1}+2 c_{2}\right] k_{\perp}^{2}+4 c_{5} k_{\|}^{2}\right)$. The probably strong relaxation of the relative rotations could damp these modes rather quickly.

\section{Conclusions and perspective}

We have investigated the influence of a passive transient or permanent network on systems with a polar dynamic active preferred direction, the latter giving rise to a second type of fluid and thus to a second velocity field. To describe the permanent and/or transient networks, we use the strain tensor as a macroscopic variable. We have elucidated the differences to the hydrodynamics of crystals for which the displacement vector is a truly hydrodynamic variable. In bio-inspired systems, the presence of a transient or permanent network is ubiquitous. We just mention the case of bacteria moving on and/or in an agar gel (Watanabe et al. 2002; Yamazaki et al. 2005)

When writing down the couplings, we pointed out those that are specific for a dynamic preferred direction and also commented on those that are unchanged with respect to the case of static order in the form of a vector or a director. For the macroscopic description of a system including a network as well as a second velocity, we find intricate cross-coupling terms between the active polar order and transient elasticity. These give rise to a combined relaxation of the polar order parameter and strains as well as to an additional propagating mode in the sound spectrum due to the reversible coupling between elasticity and polar order. Relative rotations could, depending on their relaxation times, lead to additional sound-like excitations. Or to put it differently, a sufficiently slow relaxation of relative rotations will lead to propagating modes due to the reversible coupling of relative rotations to flow, polar order, and elasticity.

In an appendix, we have demonstrated that systems that have a polar dynamic preferred direction as well as a transient or permanent network and are, in addition, chiral, possess novel reversible cross-coupling terms that lead in particular to reversible heat and concentration currents. We also note that chirality opens up additional dissipation channels.
The present general description of active dynamic polar order with a network should be applicable to systems, where two different velocities, for the active and the passive part, in addition to a gel, are important. Among those, one can think of bio-convection of bacteria colonies in a nonNewtonian solvent background or in general of bacteria and cells moving in non-Newtonian fluids as they are common in biological systems on the microscale.

We are aware that the two-fluid description is rather comprehensive. Reductions and simplifications for particular systems are plausible. Active entities like bacteria and other unicellular organisms, propelled microtubules, insects, etc. are often functioning on or in close proximity of a substrate and in an effective contact with it, which can eventually make the momentum conservation obsolete. In such cases, the background velocity can be left out and only the active velocity enters the description (Svenšek et al. 2013). A possible inclusion of a gel-like background is nevertheless sensible also in these cases. Moreover, sometimes even when momentum is conserved, the passive fluid is exempt from consideration, like, e.g., in collective dynamics of birds, fish, flying insects, etc. There the coupling to the background fluid does exist but is normally neglected, typically because the "driving" force is sophisticated and rather autonomous. These systems are driven far from equilibrium, due to the existence of sensing and nervous processing/control. Their properties are also not necessarily due to local interactions (Pearce et al. 2014).

As a challenge for future work, we leave the question to what extent the approach presented here must be modified to macroscopically describe active polar gels, where the strain formation and relaxation is an active process.

Acknowledgments Open access funding provided by Max Planck Institute for Polymer Research. H.R.B. and H.P. acknowledge partial support by the Deutsche Forschungsgemeinschaft through the Schwerpunktsprogramm SPP 1681 'Feldgesteuerte Partikel-MatrixWechselwirkungen: Erzeugung, skalenübergreifende Modellierung und Anwendung magnetischer Hybridmaterialien'. D.S. acknowledges the support of the Slovenian Research Agency, Grant No. P1-0099: Physics of soft matter, surfaces, and nanostructures.

Open Access This article is distributed under the terms of the Creative Commons Attribution 4.0 International License (http:// creativecommons.org/licenses/by/4.0/), which permits unrestricted use, distribution, and reproduction in any medium, provided you give appropriate credit to the original author(s) and the source, provide a link to the Creative Commons license, and indicate if changes were made.

\section{Appendix A: chiral additions}

In chiral systems, the inversion symmetry in space is broken. In hydrodynamics, the most efficient way of dealing with that is the introduction of a pseudoscalar quantity, $q_{0}$, that changes sign under inversion. Such a quantity exists either 
because the units or molecules are chiral by themselves and have a specific handedness, or the order parameter structure is complicated enough to allow for a $q_{0}$. In the former case, the handedness of the system is fixed, while in the latter, generally, both types of handedness are possible and energetically equivalent (ambidextrous chirality) (Brand et al. 2002; Brand and Pleiner 2010). The present case is of the first type and the handedness can originate from the active as well as the passive part.

For the energy density, we have chiral contributions that add to the sum of energies, Eq. 16,

$$
\begin{aligned}
\epsilon_{\text {chir }}= & q_{0} \tilde{K}_{2} \mathbf{f} \cdot(\nabla \times \mathbf{F}) \\
& -q_{0} \mathbf{f} \cdot(\nabla \times \mathbf{F})\left(\tau_{\phi} \delta \phi+\tau_{\sigma} \delta \sigma+\tau_{\rho} \delta \rho+\tau_{\varepsilon} \varepsilon_{i i}\right) \\
& +q_{0} \tau^{\Omega} f_{j} f_{m} \epsilon_{i m k} \tilde{\Omega}_{i} \nabla_{j} F_{k}
\end{aligned}
$$

describing first linear twist, responsible for the helical structure of the preferred direction to be the energetic minimum state (de Gennes 1975). Second are the static Lehmann-type couplings (Brand and Pleiner 1988, 2001) to scalar variables and to elastic compressional deformations. The final term is operational only for distorted helical structures. This last contribution is a higher order gradient term. It also exists in the usual active polar systems without viscoelastic effects (Brand et al. 2013).

The energy $\epsilon_{\text {chir }}$ is the same as for a passive chiral nematic elastomer (replacing $f_{i}$ by the director $n_{i}$ everywhere).

In the dissipation, the chiral additions to Eq. 33 are, neglecting some higher order gradient terms,

$$
\begin{aligned}
R_{\text {chir }}= & q_{0} f_{i} \epsilon_{i j k}\left(\psi_{j}^{(1)} \Sigma_{k}+\psi_{j}^{(2)} h_{k}\right)+q_{0} \psi_{i j}^{(3)} \Xi_{i j} \\
& +q_{0} \kappa_{i j k}^{\|}\left(\zeta_{1}^{A} \Sigma_{i}+\zeta_{2}^{A} h_{i}\right) A_{j k} \\
& +q_{0}\left(\zeta_{\|}^{A} \kappa_{j k l m}^{\|}+\zeta_{\perp}^{A} \kappa_{j k l m}^{\perp}\right) A_{j k} \Xi_{l m}
\end{aligned}
$$

where

$$
\begin{aligned}
\psi_{j}^{(1,2)} & \equiv \psi_{1,2}^{T} \nabla_{j} T+\psi_{1,2}^{\Pi} \nabla_{j} \Pi^{\prime}+\psi_{1,2}^{m} \nabla_{j} m \\
\psi_{i j}^{(3)} & \equiv \sum_{B}\left(\psi_{3 \|}^{B} \kappa_{k i j}^{\|}+\psi_{3 \perp}^{B} \kappa_{k i j}^{\perp}\right) \nabla_{k} B
\end{aligned}
$$

for $B=T, \Pi^{\prime}, m$ with

$$
\begin{aligned}
\kappa_{i j k}^{\|}= & \frac{1}{2}\left(f_{l} f_{k} \epsilon_{i j l}+f_{l} f_{j} \epsilon_{i k l}\right) \\
\kappa_{i j k}^{\perp}= & \frac{1}{2}\left(\delta_{l k}^{\perp} \epsilon_{i j l}+\delta_{l j}^{\perp} \epsilon_{i k l}\right) \\
\kappa_{j k l m}^{\|}= & \frac{1}{4} f_{i}\left(f_{k} f_{m} \epsilon_{i j l}+f_{l} f_{j} \epsilon_{i k m}\right. \\
& \left.\quad+f_{j} f_{m} \epsilon_{i k l}+f_{l} f_{k} \epsilon_{i j m}\right) \\
\kappa_{j k l m}^{\perp}= & \frac{1}{4} f_{i}\left(\delta_{k m}^{\perp} \epsilon_{i j l}+\delta_{j l}^{\perp} \epsilon_{i k m}+\delta_{j m}^{\perp} \epsilon_{i k l}+\delta_{k l}^{\perp} \epsilon_{i j m}\right)
\end{aligned}
$$

The first line contains generalized dynamic Lehmann contributions (Brand and Pleiner 1988, 2001; Svenšek et al. 2008) that relate gradients of temperature, osmotic pressure, and order deviations with relative rotations $\left(\psi_{i}^{(1)}\right)$, reorientations of the preferred direction $\left(\psi_{i}^{(2)}\right)$, and relaxing strains $\left(\psi_{i j}^{(3)}\right)$. Such terms also exist in passive systems. The chiral couplings of flow to relative rotations $\left(\sim \zeta_{1}^{A}\right)$, to reorientations of the preferred direction $\left(\sim \zeta_{2}^{A}\right)$, and to relaxing strains $\left(\sim \zeta_{\|, \perp}^{A}\right)$ are specific for a dynamic order and do not exist in passive systems.

For the chiral additions to the reversible currents, we first have

$$
\begin{aligned}
j_{i}^{\sigma R c}= & q_{0} g_{i j k}^{(1)} A_{j k}+q_{0} g_{i j k l m}^{(2)} f_{k} \nabla_{l} \Xi_{j m} \\
j_{i}^{\Phi R c}= & q_{0} g_{i j k}^{(3)} A_{j k}+q_{0} g_{i j k l m}^{(4)} f_{k} \nabla_{l} \Xi_{j m} \\
\sigma_{i j}^{R c}= & -q_{0} g_{k i j}^{(1)} \nabla_{k} T-q_{0} g_{k i j}^{(3)} \nabla_{k} \Pi^{\prime} \\
W_{i j}^{R c}= & q_{0} \nabla_{l}\left[g_{m i k l j}^{(2)} f_{k} \nabla_{m} T\right] \\
& +q_{0} \nabla_{l}\left[g_{m i k l j}^{(4)} f_{k} \nabla_{m} \Pi^{\prime}\right] \\
& +q_{0} \kappa_{k i j}^{\|}\left(g^{(5)} \Sigma_{k}+g^{(6)} h_{k}\right) \\
Z_{i}^{R c}= & -q_{0} g^{(5)} \kappa_{i j k}^{\|} \Xi_{j k} \\
Y_{i}^{R c}= & -q_{0} g^{(6)} \kappa_{i j k}^{\|} \Xi_{j k}
\end{aligned}
$$

where for $n=1,3$

$g_{i j k}^{(n)}=g_{n}^{\|} \kappa_{i j k}^{\|}+g_{n}^{\perp} \kappa_{i j k}^{\perp}$

and for $n=2,4$

$$
\begin{aligned}
g_{i j k l m}^{(n)}= & g^{n 1}\left(\varepsilon_{i j k} \delta_{l m}^{\perp}+\varepsilon_{i m k} \delta_{l j}^{\perp}\right) \\
& +g^{n 2}\left(\varepsilon_{j k l} \delta_{i m}^{\perp}+\varepsilon_{m k l} \delta_{i j}^{\perp}\right) \\
& +g^{n 3} \varepsilon_{i k l} \delta_{j m}^{\perp}+g^{n 4} \varepsilon_{i k l} f_{j} f_{m} \\
& +g^{n 5}\left(\varepsilon_{i j k} f_{l} f_{m}+\varepsilon_{i m k} f_{l} f_{j}\right) \\
& +g^{n 6}\left(\varepsilon_{i j l} f_{k} f_{m}+\varepsilon_{i m l} f_{k} f_{j}\right) \\
& +g^{n 7}\left(\varepsilon_{j k l} f_{i} f_{m}+\varepsilon_{m k l} f_{i} f_{j}\right)
\end{aligned}
$$

describing mutual couplings of elasticity with temperature, osmotic pressure, relative rotations, and reorientations of the preferred direction $\left(g_{i j k l m}^{(2)}, g_{i j k l m}^{(4)}, g^{(5)}, g^{(6)}\right.$, respectively), while the flow couplings $\left(g_{i j k}^{(1,3)}\right)$ are known already from chiral active polar systems with a simple fluid background (Brand et al. 2013).

In the case of a crystal (cf. Appendix B), these chiral additions to the reversible currents take the simpler form

$$
\begin{aligned}
j_{i}^{\sigma R c}= & \cdots+q_{0} g_{2} \epsilon_{i j k} f_{k} \nabla_{l} \Xi_{j l} \\
j_{i}^{\Phi R c}= & \cdots+q_{0} g_{4} \epsilon_{i j k} f_{k} \nabla_{l} \Xi_{j l} \\
W_{i j}^{R c}= & \cdots+\frac{1}{2} q_{0} g_{2}\left(\epsilon_{i k l} \nabla_{j}\left[f_{l} \nabla_{k} T\right]+\epsilon_{j k l} \nabla_{i}\left[f_{l} \nabla_{k} T\right]\right) \\
& +\frac{1}{2} q_{0} g_{4}\left(\epsilon_{i k l} \nabla_{j}\left[f_{l} \nabla_{k} \Pi^{\prime}\right]+\epsilon_{j k l} \nabla_{i}\left[f_{l} \nabla_{k} \Pi^{\prime}\right]\right)
\end{aligned}
$$

Thus, we see that the seven coefficients involved in the reversible coupling of temperature and concentration gradients to elasticity diffusion for gels, rubbers, and disordered 
solids reduce to only one independent contribution in the case of crystals.

Second, there are self-coupling terms, a new one involving relative rotations $\left(d_{4}\right)$, in addition to that of flow $\left(d_{3}\right)$ and of reorientations of the preferred direction $\left(d_{5}\right)$, known before. The appropriate self-couplings of the thermal $\left(d_{1}\right)$ and soluble $\left(d_{2}\right)$ degree of freedom are surface terms

$$
\begin{aligned}
j_{i}^{\sigma R c n} & =q_{0} d_{1} \epsilon_{i j k} f_{k} \nabla_{j} T \\
j_{i}^{\Phi R c n} & =q_{0} d_{2} \epsilon_{i j k} f_{k} \nabla_{j} \Pi^{\prime} \\
\sigma_{i j}^{R c n} & =q_{0}\left(d_{3}^{\|} \kappa_{i j l m}^{\|}+d_{3}^{\perp} \kappa_{i j l m}^{\perp}\right) A_{l m} \\
W_{i j}^{R c} & =q_{0}\left(d_{6}^{\|} \kappa_{i j l m}^{\|}+d_{6}^{\perp} \kappa_{i j l m}^{\perp}\right) \Xi_{l m} \\
Z_{i}^{R c n} & =q_{0} d_{4} \epsilon_{i j k} f_{k} \Sigma_{j} \\
Y_{i}^{R c n} & =q_{0} d_{5} \epsilon_{i j k} f_{k} h_{j}
\end{aligned}
$$

These terms do not have a counterpart, but are nilpotent in the entropy production. They are either associated with local distortions of the helical structure or vanish when averaged over many pitch lengths.

The reversible contributions to the stress tensor in Eq. 81 have been found first for the superfluid $A$ phase of ${ }^{3} \mathrm{He}$ (Liu 1976). They also arise for ${ }^{3} \mathrm{He}-A_{1}$ (Pleiner and Graham 1976; Liu 1979), ${ }^{3} \mathrm{He}-A$ in high magnetic fields (Brand and Pleiner 1981) and in ${ }^{3} \mathrm{He}-B$ in high magnetic fields (Pleiner and Brand 1983). They always contain hydrodynamic contributions from the memory matrix (Brand et al. 1979; Brand and Pleiner 1982). These reversible contributions to the stress tensor also arise for uniaxial magnetic gels (Bohlius et al. 2004) as well as for ferrocholesterics in a magnetic fields (Jarkova et al. 2001). In the latter case, one obtains eight independent coefficients due to the lower symmetry of the system (Jarkova et al. 2001). Analyzing the structure of these terms in detail one arrives at the conclusion that for these contributions to arise one needs either a preferred direction, which is odd under time reversal and even under parity (Liu 1976, 1979; Pleiner and Graham 1976; Pleiner and Brand 1983; Brand and Pleiner 1981, 1982; Brand et al. 1979; Bohlius et al. 2004) or a preferred direction, which is odd under time reversal as well as odd under parity, but has in addition a pseudoscalar quantity, as is the case studied here and in ref. Brand et al. (2013). In all cases, there are reversible contributions from the memory matrix.

\section{Appendix B: dynamic processes associated with the strain tensor in gels and rubbers versus dynamic effects associated with the displacement field in crystals}

In the bulk part of this paper, we have taken the six components of the strain tensor $\varepsilon_{i j}$ as independent macroscopic variables. Such an approach is appropriate for gels, rubbers, transient networks, and also for solids with defects.

The purpose of this appendix is to show how these results reduce to those of a crystal, which is described by using the displacement field $\vec{u}$ as strictly hydrodynamic variables (Martin et al. 1972). Since we deal here with linear elasticity the relation between the strain tensor and the displacement field simply reads $\varepsilon_{i j}=\frac{1}{2}\left(\nabla_{i} u_{j}+\nabla_{j} u_{i}\right)$ and certain compatibility conditions guaranteeing the existence of a displacement field are satisfied. For a detailed discussion of the relation between the displacement field and the nonlinear strain tensor in the Eulerian picture, we refer to the discussion in the Appendix of ref. Pleiner et al. (2000).

For the static aspects the discussion from "Statics" section remains unchanged when making the replacement $\varepsilon_{i j}=\frac{1}{2}\left(\nabla_{i} u_{j}+\nabla_{j} u_{i}\right)$ given above.

For the dynamic aspects, the situation is different. While the use of the thermodynamic conjugate $\Xi_{i j}$ can be taken over unchanged for reversible and irreversible currents as well as for the dissipation function, this is different for its gradients for which we had in the bulk part $\nabla_{i} \Xi_{j k}$, without any restriction for the three indices $i, j$ and $k$. Since the displacement field is a truly hydrodynamic variable in a crystal, only the expression $\nabla_{k} \Xi_{j k}$ enters the hydrodynamic description (Martin et al. 1972). In addition, there is no term $\sim \Xi_{i j}$ without gradients in the dissipation function, since the displacement field is truly hydrodynamic. Therefore, the last line of Eq. 33 vanishes completely. Thus, we have for the simplified dissipation function in a crystal for the parts associated with the displacement field

$$
\begin{aligned}
2 R= & \cdots+\xi_{i j}\left(\nabla_{k} \Xi_{i k}\right)\left(\nabla_{l} \Xi_{j l}\right)+2 \mu_{i j k}^{\Xi} A_{i j} \nabla_{l} \Xi_{k l} \\
& +2 \xi_{i j}^{T}\left(\nabla_{i} T\right)\left(\nabla_{k} \Xi_{j k}\right)+2 \xi_{i j}^{\Pi}\left(\nabla_{i} \Pi^{\prime}\right)\left(\nabla_{k} \Xi_{j k}\right)
\end{aligned}
$$

The second rank tensors have the form of Eq. 22 and carry two coefficients each, while the third rank tensor is of the form Eq. 23 with three coefficients.

Using the strain tensor instead, there are material tensors of rank four, five, and six involved in Eq. 33, carrying much more coefficients. In particular, we find for

$R_{5}=\frac{1}{2} \mu_{i j k l m}^{\Xi} A_{i j} \nabla_{k} \Xi_{l m}$

12 coefficients using the symmetries $i \leftrightarrow j$ and $l \leftrightarrow m$,

$$
\begin{aligned}
& \mu_{i j k l m}^{\Xi}=\mu_{1}^{\Xi} f_{k} \delta_{i j}^{\perp} \delta_{l m}^{\perp}+\mu_{2}^{\Xi}\left(\delta_{i l}^{\perp} \delta_{j m}^{\perp}+\delta_{j l}^{\perp} \delta_{i m}^{\perp}\right) f_{k} \\
& +\mu_{3}^{\Xi} \delta_{l m}^{\perp}\left(\delta_{k j}^{\perp} f_{i}+\delta_{k i}^{\perp} f_{j}\right)+\mu_{4}^{\Xi} \delta_{i j}^{\perp}\left(\delta_{k l}^{\perp} f_{m}+\delta_{k m}^{\perp} f_{l}\right) \\
& +\mu_{5}^{\Xi}\left[\left(\delta_{k l}^{\perp} \delta_{j m}^{\perp}+\delta_{k m}^{\perp} \delta_{j l}^{\perp}\right) f_{i}+\left(\delta_{k l}^{\perp} \delta_{i m}^{\perp}+\delta_{k m}^{\perp} \delta_{i l}^{\perp}\right) f_{j}\right] \\
& +\mu_{6}^{\Xi}\left[\left(\delta_{i k}^{\perp} \delta_{j l}^{\perp}+\delta_{j k}^{\perp} \delta_{i l}^{\perp}\right) f_{m}+\left(\delta_{i k}^{\perp} \delta_{j m}^{\perp}+\delta_{j k}^{\perp} \delta_{i m}^{\perp}\right) f_{l}\right] \\
& +\mu_{7}^{\Xi} \delta_{i j}^{\perp} f_{k} f_{l} f_{m}+\mu_{8}^{\Xi} \delta_{l m}^{\perp} f_{i} f_{j} f_{k}+\mu_{9}^{\Xi} f_{i} f_{j} f_{k} f_{l} f_{m} \\
& +\mu_{10}^{\Xi}\left(\delta_{i k}^{\perp} f_{j}+\delta_{j k}^{\perp} f_{i}\right) f_{l} f_{m}+\mu_{11}^{\Xi}\left(\delta_{k l}^{\perp} f_{m}+\delta_{k m}^{\perp} f_{l}\right) f_{i} f_{j}(92) \\
& +\mu_{12}^{\Xi}\left(\delta_{i l}^{\perp} f_{m} f_{j}+\delta_{i m}^{\perp} f_{l} f_{j}+\delta_{j l}^{\perp} f_{m} f_{i}+\delta_{j m}^{\perp} f_{l} f_{i}\right) f_{k}
\end{aligned}
$$


and for

$R_{6}=\frac{1}{2} \xi_{i j k l p q}\left(\nabla_{k} \Xi_{i p}\right)\left(\nabla_{l} \Xi_{j q}\right)$

16 coefficients using the symmetries $k \leftrightarrow l, i \leftrightarrow p$, and $j \leftrightarrow q$, as well as $i \leftrightarrow j \wedge p \leftrightarrow q$

$$
\begin{aligned}
& \xi_{i j k l p q}=\xi_{1} \delta_{i p}^{\perp} \delta_{j q}^{\perp} \delta_{l k}^{\perp} \\
& +\xi_{2}\left(\delta_{i j}^{\perp} \delta_{p q}^{\perp}+\delta_{i q}^{\perp} \delta_{j p}^{\perp}\right) \delta_{l k}^{\perp} \\
& +\xi_{3}\left(\left[\delta_{i k}^{\perp} \delta_{l p}^{\perp}+\delta_{i l}^{\perp} \delta_{p k}^{\perp}\right] \delta_{j q}^{\perp}+\left[\delta_{j k}^{\perp} \delta_{q l}^{\perp}+\delta_{j l}^{\perp} \delta_{q k}^{\perp}\right] \delta_{i p}^{\perp}\right) \\
& +\xi_{4}\left(\delta_{i j}^{\perp} \delta_{l p}^{\perp} \delta_{q k}^{\perp}+\delta_{p j}^{\perp} \delta_{l i}^{\perp} \delta_{q k}^{\perp}+\delta_{i q}^{\perp} \delta_{l p}^{\perp} \delta_{j k}^{\perp}+\delta_{p q}^{\perp} \delta_{l i}^{\perp} \delta_{j k}^{\perp}\right. \\
& \left.+\delta_{i j}^{\perp} \delta_{k p}^{\perp} \delta_{q l}^{\perp}+\delta_{p j}^{\perp} \delta_{k i}^{\perp} \delta_{q l}^{\perp}+\delta_{i q}^{\perp} \delta_{k p}^{\perp} \delta_{j l}^{\perp}+\delta_{p q}^{\perp} \delta_{k i}^{\perp} \delta_{j l}^{\perp}\right) \\
& +\xi_{5}\left(f_{i} f_{p} \delta_{j q}^{\perp} \delta_{l k}^{\perp}+\delta_{i p}^{\perp} f_{j} f_{q} \delta_{l k}^{\perp}\right)+\xi_{6} \delta_{i p}^{\perp} \delta_{j q}^{\perp} f_{l} f_{k} \\
& +\xi_{7}\left(\delta_{i j}^{\perp} \delta_{p q}^{\perp}+\delta_{i q}^{\perp} \delta_{j p}^{\perp}\right) f_{l} f_{k} \\
& +\xi_{8}\left(f_{i} f_{j} \delta_{p q}^{\perp}+f_{i} f_{q} \delta_{j p}^{\perp}+\delta_{i j}^{\perp} f_{p} f_{q}+\delta_{i q}^{\perp} f_{j} f_{p}\right) \delta_{l k}^{\perp} \\
& +\xi_{9}\left(\left[f_{i} f_{k} \delta_{l p}^{\perp}+f_{i} f_{l} \delta_{p k}^{\perp}+\delta_{i k}^{\perp} f_{l} f_{p}+\delta_{i l}^{\perp} f_{p} f_{k}\right] \delta_{j q}^{\perp}\right. \\
& \left.+\left[f_{j} f_{k} \delta_{q l}^{\perp}+f_{j} f_{l} \delta_{q k}^{\perp}+\delta_{j k}^{\perp} f_{q} f_{l}+\delta_{j l}^{\perp} f_{q} f_{k}\right] \delta_{i p}^{\perp}\right) \\
& +\xi_{10}\left(\left[\delta_{i k}^{\perp} \delta_{l p}^{\perp}+\delta_{i l}^{\perp} \delta_{p k}^{\perp}\right] f_{j} f_{q}+\left[\delta_{j k}^{\perp} \delta_{q l}^{\perp}+\delta_{j l}^{\perp} \delta_{q k}^{\perp}\right] f_{i} f_{p}\right) \\
& +\xi_{11}\left(f_{i} f_{j} \delta_{l p}^{\perp} \delta_{q k}^{\perp}+\delta_{i j}^{\perp} f_{l} f_{p} \delta_{q k}^{\perp}+\delta_{i j}^{\perp} \delta_{l p}^{\perp} f_{q} f_{k}\right. \\
& +f_{p} f_{j} \delta_{l i}^{\perp} \delta_{q k}^{\perp}+\delta_{p j}^{\perp} f_{i} f_{l} \delta_{q k}^{\perp}+\delta_{p j}^{\perp} \delta_{l i}^{\perp} f_{k} f_{q} \\
& +f_{i} f_{q} \delta_{l p}^{\perp} \delta_{j k}^{\perp}+\delta_{i q}^{\perp} f_{l} f_{p} \delta_{j k}^{\perp}+\delta_{i q}^{\perp} \delta_{l p}^{\perp} f_{j} f_{k} \\
& +f_{p} f_{q} \delta_{l i}^{\perp} \delta_{j k}^{\perp}+\delta_{p q}^{\perp} f_{i} f_{l} \delta_{j k}^{\perp}+\delta_{p q}^{\perp} \delta_{l i}^{\perp} f_{j} f_{k} \\
& +f_{i} f_{j} \delta_{k p}^{\perp} \delta_{q l}^{\perp}+\delta_{i j}^{\perp} f_{k} f_{p} \delta_{q l}^{\perp}+\delta_{i j}^{\perp} \delta_{k p}^{\perp} f_{l} f_{q} \\
& +f_{p} f_{j} \delta_{k i}^{\perp} \delta_{q l}^{\perp}+\delta_{p j}^{\perp} f_{i} f_{k} \delta_{q l}^{\perp}+\delta_{p j}^{\perp} \delta_{k i}^{\perp} f_{l} f_{q} \\
& +f_{i} f_{q} \delta_{k p}^{\perp} \delta_{j l}^{\perp}+\delta_{i q}^{\perp} f_{k} f_{p} \delta_{j l}^{\perp}+\delta_{i q}^{\perp} \delta_{k p}^{\perp} f_{j} f_{l} \\
& \left.+f_{p} f_{q} \delta_{k i}^{\perp} \delta_{j l}^{\perp}+\delta_{p q}^{\perp} f_{k} f_{i} \delta_{j l}^{\perp}+\delta_{p q}^{\perp} \delta_{k i}^{\perp} f_{j} f_{l}\right) \\
& +\xi_{12} \delta_{l k}^{\perp} f_{i} f_{p} f_{j} f_{q} \\
& +\xi_{13}\left(\delta_{j q}^{\perp} f_{i} f_{p} f_{l} f_{k}+\delta_{i p}^{\perp} f_{j} f_{q} f_{l} f_{k}\right) \\
& +\xi_{14}\left(\delta_{p q}^{\perp} f_{i} f_{j}+\delta_{j p}^{\perp} f_{i} f_{q}+\delta_{i j}^{\perp} f_{p} f_{q}+\delta_{i q}^{\perp} f_{j} f_{p}\right) f_{l} f_{k} \\
& +\xi_{15}\left(\left[\delta_{k i}^{\perp} f_{p} f_{l}+\delta_{k p}^{\perp} f_{i} f_{l}+\delta_{l i}^{\perp} f_{p} f_{k}+\delta_{l p}^{\perp} f_{i} f_{k}\right] f_{j} f_{q}\right. \\
& \left.+\left[\delta_{q l}^{\perp} f_{j} f_{k}+\delta_{j l}^{\perp} f_{q} f_{k}+\delta_{j k}^{\perp} f_{l} f_{q}+\delta_{q k}^{\perp} f_{j} f_{l}\right] f_{i} f_{p}\right) \\
& +\xi_{16} f_{i} f_{j} f_{k} f_{l} f_{p} f_{q}
\end{aligned}
$$

For the reversible currents containing gradients of the elastic stress tensor, we obtain for crystals the slightly simplified expressions

$$
\begin{aligned}
Y_{i}^{R}= & \cdots+\delta_{i j}^{\perp} \beta^{W} \nabla_{k} \Xi_{j k} \\
X^{R}= & \cdots+\beta_{1}^{W} f_{i} \nabla_{k} \Xi_{i k} \\
Z_{i}^{R}= & \cdots+\beta^{\Omega} \delta_{i j}^{\perp} \nabla_{k} \Xi_{j k} \\
W_{i j}^{R}= & \frac{1}{2} \beta_{1}^{W}\left(f_{i} \nabla_{j} m+f_{j} \nabla_{i} m\right) \\
& +\frac{1}{2}\left(\delta_{i k}^{\perp} \nabla_{j}+\delta_{j k}^{\perp} \nabla_{i}\right)\left(\beta^{\Omega} \Sigma_{k}-\beta^{W} h_{k}\right)
\end{aligned}
$$

Thus, instead of obtaining second rank tensors for the case of the strain field as a variable, we get a scalar coefficient each for the case of a displacement field as a truly hydrodynamic variable.

\section{References}

Aditi Simha R, Ramaswamy S (2002) Hydrodynamic fluctuations and instabilities in ordered suspensions of self-propelled particles. Phys Rev Lett 89:058101

Akhmediev N, Soto-Crespo JM, Brand HR (2013) Dissipative solitons with energy and matter flows: fundamental building blocks for the world of living organisms. Phys Lett A 377:968-974

Ballerini M, Cabibbo N, Candeller R, Cavagna A, Cisbani E, Giardina I, Lecomte V, Orlandi A, Parisi G, Procaccini A, Viale M, Zdravkovic V (2008) Interaction ruling animal collective behavior depends on topological rather than metric distance: evidence from a field study. Proc Natl Acad Sci USA 105:1232-1237

Bird RB, Armstrong RC, Hassager O (1977) Dynamics of polymeric liquids, vol 1. Wiley, New York

Bohlius S, Brand HR, Pleiner H (2004) Macroscopic dynamics of uniaxial magnetic gels. Phys Rev E 70:061411

Brand H, Pleiner H (1981) Linearized hydrodynamics of ${ }^{3} \mathrm{He}-\mathrm{A}$ in high magnetic fields. J Phys C 14:97-103

Brand H, Pleiner H (1982) Linearized hydrodynamics of ${ }^{3} \mathrm{He}-\mathrm{A}_{1}$, correlation functions and hydrodynamic parameters. J Phys (Paris) 43:369-380

Brand HR, Pleiner H (1988) New theoretical results for the Lehmann effect in cholesteric liquid crystals. Phys Rev A 37:2736-2738

Brand HR, Pleiner H (1994) Electrohydrodynamics of nematic liquid crystalline elastomers. Physica A 208:359-372

Brand HR, Pleiner H (2001) In encyclopedia of materials: science and technology. Elsevier 5:1214

Brand HR, Pleiner H (2010) Macroscopic behavior of non-polar tetrahedratic nematic liquid crystals. Eur Phys J E 31:37-50

Brand H, Dörfle M, Graham R (1979) Hydrodynamic parameters and correlation functions of superfluid ${ }^{3} \mathrm{He}$. Ann Phys (NY) 119:434479

Brand HR, Pleiner H, Cladis PE (2002) Flow properties of the optically isotropic tetrahedratic phase. Eur Phys J E 7:163-166

Brand HR, Pleiner H, Ziebert F (2006) Macroscopic dynamics of polar nematic liquid crystals. Phys Rev E 74:021713

Brand HR, Cladis PE, Pleiner H (2009) Reversible macroscopic dynamics of polar nematic liquid crystals: reversible currents and their experimental consequences. Phys Rev E 79:032701

Brand HR, Pleiner P, Svenšek D (2011) Macroscopic behavior of nonpolar tetrahedratic nematic liquid crystals. Eur Phys J E 34:128

Brand HR, Pleiner H, Svenšek D (2013) Active polar two-fluid macroscopic dynamics. Eur Phys J E 36:135

Brand HR, Pleiner H, Svenšek D (2014) Reversible and dissipative macroscopic contributions to the stress tensor: active or passive? Eur Phys J E 37:83

Buhl J, Sumpter DJT, Couzin ID, Hale JJ, Despland U, Miller ER, Simpson SJ (2006) From disorder to order in marching locusts. Science 312:1402-1406

Cisneros L, Cortez R, Dombrovski C, Goldstein R, Kessler J (2007) Fluid dynamics if self-propelled microorganisms, from individuals to concentrated populations. Exp Fluids 43:737-753

Coleman BD, Noll W (1961) Foundations of linear viscoelasticity. Rev Mod Phys 33:239-249

Cross MC, Hohenberg PC (1993) Pattern-formation outside of equilibrium. Rev Mod Phys 65:851-1112

Doi M, Edwards SF (1986) The theory of polymer dynamics. Clarendon Press, Oxford 
Fu X, Tang L, Liu C, Huang J, Hwa T, Lenz P (2012) Stripe formation in bacterial systems with density-suppressed motility. Phys Rev Lett 108:198102

de Gennes PG (1975) The physics of liquid crystals. Clarendon Press, Oxford

deGennes PG (1980) In liquid crystals of one- and two-dimensional order. In: Helfrich W, Heppke G (eds). Springer, New York

Giesekus H (1966) Die Elastizität von Flüssigkeiten. Rheol Acta 5:29_ 35

Giesekus H (1982) A simple constitutive equation for polymer fluids based on the concept of deformation-dependent tensorial mobility. J Non-Newt Fluid Mech 11:69-109

Giomi L, Marchetti MC (2012) Polar patterns in active fluids. Soft Matter 8:129-139

Grmela M (2002) Lagrange hydrodynamics as extended Euler hydrodynamics: Hamiltonian and GENERIC structures. Phys Lett A 296:97-104

Hatwalne Y, Ramaswamy S, Rao M, Aditi Simha R (2004) Rheology of active-particle suspensions. Phys Rev Lett 92:118101

Jarkova E, Pleiner H, Müller HW, Fink A, Brand HR (2001) Hydrodynamics of nematic ferrofluids. Eur Phys J E 5:583-588

Johnson MW, Segalman D (1977) Model for viscoelastic fluid behavior which allows nonaffine deformation. J Non-Newt Fluid Mech 2:255-270

Johnson MW, Segalman D (1978) Model for viscoelastic fluid behavior which allows nonaffine deformation. J Rheol 22:445-446

Katz Y, Tunstrom K, Ioannou CC, Huepe C, Couzin Y (2011) Inferring the structure and dynamics of interactions in schooling fish. Proc Natl Acad Sci USA 108:18720-18725

Larson RG (1988) Constitutive equations for polymer melts and solutions. Butterworths, Boston

Liu M (1976) Comment on 'Hydrodynamics of ${ }^{3} \mathrm{He}$ in anisotropic Aphase'. Phys Rev B 13:4174-4174

Liu M (1979) Broken relative symmetry and the dynamics of the $A_{1}$ phase of ${ }^{3} \mathrm{He}$. Phys Rev Lett 43:1740-1743

Loose M, Fischer-Friedrich E, Ries J, Kruse K, Schwille P (2008) Spatial regulators for bacterial cell division into surface waves in vitro. Science 320:789-792

Lukeman R, Li Y, Edelstein-Keshet L (2010) Inferring individual rules from collective behavior. Proc Natl Acad Sci USA 107:1257612580

Maitra A, Srivastava P, Rao M, Ramaswamy S (2014) Activating membranes. Phys Rev Lett 112:258101

Marchetti MC, Joanny JF, Ramaswamy S, Liverpool TB, Prost J, Rao M, Aditi Simha R (2013) Hydrodynamics of soft active matter. Rev Mod Phys 85:1143

Martin PC, Parodi O, Pershan PS (1972) Unified hydrodynamic theory for crystals, liquid crystals and normal fluids. Phys Rev A 6:24012424

Matsushita M, Wakita J, Itoh H, Ràfols I, Matsuyama T, Sakaguchi $\mathrm{H}$, Mimura M (1998) Interface growth and pattern formation in bacterial colonies. Physica A 249:517-524

Matsushita M, Wakita J, Itoh H, Watanabe K, Arai T, Matsuyama T, Sakaguchi H, Mimura M (1999) Formation of colony patterns by a bacterial cell population. Physica A 274:190-199

Menzel AM, Pleiner H, Brand HR (2007) Nonlinear relative rotations in liquid crystalline elastomers. J Chem Phys 126:234901

Menzel AM, Pleiner H, Brand HR (2009a) On the nonlinear stressstrain behavior of nematic elastomers-materials of two coupled preferred directions. J Appl Phys 105:013593

Menzel AM, Pleiner H, Brand HR (2009b) Response of prestretched nematic elastomers to external fields. Eur Phys J E 30:371377

Moriyama O, Matsushita M (1995) Simple model for target patterns and spiral waves. J Phys Soc Jpn 64:1081-1084
Müller O, Liu M, Pleiner H, Brand HR (2016a) Transient elasticity and polymeric fluids: small amplitude deformations. Phys Rev E 93:023113

Müller O, Liu M, Pleiner H, Brand HR (2016b) Transient elasticity and the rheology of polymeric fluids with large amplitude deformations. Phys Rev E 93:023114

Nédélec F, Surrey T, Maggs AC, Leibler S (1997) Self-organization of microtubules and motors. Nature 389:305-308

Oldroyd JG (1950) On the formulation of rheological equations of state. Proc Roy Soc A 200:523-541

Oldroyd JG (1961) The hydrodynamics of materials whose rheological problems are complicated. Rheol Acta 1:337-344

Parrish JK, Edelstein-Keshet L (1999) Complexity, pattern, and evolutionary trade-offs in animal aggregation. Science 284:99-101

Pearce DJG, Miller AM, Rowlands G, Turner MS (2014) Role of projection in the control of bird flocks. Proc Natl Acad Sci USA 111:10422-10426

Pleiner H, Graham R (1976) Macroscopic dynamics of ${ }^{3} \mathrm{He}$ in magnetic $A_{1}$ phase. J Phys C 9:4109-4130

Pleiner H, Brand H (1983) Linearized hydrodynamics of superfluid ${ }^{3} \mathrm{He}-\mathrm{B}$ in high magnetic fields. Phys Rev B 28:3782-3792

Pleiner H, Brand HR (1991) Macroscopic dynamic equations for nematic liquid crystalline side-chain polymers. Mol Cryst Liq Cryst 199:407-418

Pleiner H, Brand HR (1996) In pattern formation in liquid crystals. In: Buka A, Kramer L (eds). Springer, New York, p 15

Pleiner H, Harden JL (2003) In nonlinear problems of continuum mechanics, special issue of notices of universities. South of Russia. Natural sciences, p 46 and arXiv:cond-mat/0404134

Pleiner H, Liu M, Brand HR (2000) The structure of convective nonlinearities in polymer rheology. Rheol Acta 39:560-565

Pleiner H, Liu M, Brand HR (2002) Convective nonlinearities for the orientational tensor order parameter in polymeric systems. Rheol Acta 41:375-382

Pleiner H, Liu M, Brand HR (2004) Nonlinear fluid dynamics description of non-Newtonian fluids. Rheol Acta 43:502-508

Pleiner H, Liu M, Brand HR (2005) In IMA volume in mathematics and its applications. In: Calderer M-CT, Terentjev EM (eds), vol 141. Springer, Berlin, p 99

Rogez D, Martinoty P (2011) Mechanical properties of monodomain nematic side-chain liquid-crystalline elastomers with homeotropic and in-plane orientation of the director. Eur Phys J E 34:69

Schaller V, Weber C, Semmrich C, Frey E, Bausch AR (2010) Polar patterns of driven filaments. Nature 467:73-77

Schaller V, Weber CA, Hammerich B, Frey E, Bausch AR (2011) Frozen steady states in active systems. Proc Natl Acad Sci USA 108:19183-19188

Surrey T, Nédélec F, Leibler S, Karsenti E (2001) Physical properties determining self-organization of motors and microtubules. Science 292:1167-1171

Svenšek D, Pleiner H, Brand HR (2008) Inverse Lehmann effects can be used as a microscopic pump. Phys Rev E 78:021703

Svenšek D, Pleiner H, Brand HR (2013) Collective Stop-and-Go dynamics of active bacteria swarms. Phys Rev Lett 111:228101

Temmen H, Pleiner H, Liu M, Brand HR (2000) Convective nonlinearity in non-Newtonian fluids. Phys Rev Lett 84:3228-3231

Temmen H, Pleiner H, Liu M, Brand HR (2001) Comment on 'Convective nonlinearity in non-Newtonian fluids' - Temmen et al, reply. Phys Rev Lett 86:745-745

Truesdell C, Noll W (1965) The non-linear field theories of mechanics. Springer, Berlin/New York

Urayama K, Mashita R, Kobayashi I, Takigawa T (2007) Stretchinginduced director rotation in thin films of liquid crystal elastomers with homeotropic alignment. Macromol 40:7665-7670 
Vicsek T, Czirók A, Ben-Jacob E, Cohen I, Shochet O (1995) Novel type of phase transition in a system of self-driven particles. Phys Rev Lett 75:1226-1229

Watanabe K, Wakita J, Itoh H, Shimada H, Kurosu S, Ikeda T, Yamazaki Y, Matsuyama T, Matsushita M (2002) Dynamical proeprties of transient spatio-temporal patterns in bacterial colony of Proteus mirabilis. J Phys Soc Jpn 71:650-656
Yamazaki Y, Ikeda T, Shimada H, Hiramatsu F, Kobayashi N, Wakita J, Itoh H, Kurosu S, Nakatsuchi M, Matsuyama T, Matsushita M (2005) Periodic growth of bacterial colonies. Physica D 205:136153

Zhang HP, Be'er A, Florin EL, Swinney HL (2010) Collective motion and density fluctuations in bacterial colonies. Proc Natl Acad Sci USA 107:13626-13630 